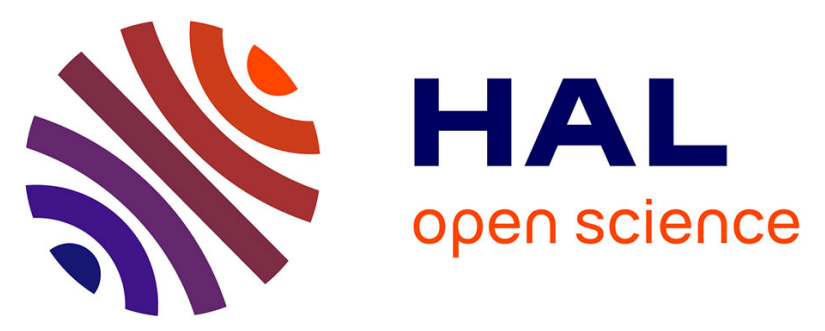

\title{
Microwell Array integrating Nanoelectrodes for Coupled Opto-electrochemical Monitorings of Single Mitochondria
}

Venkata Suresh Vajrala, Fadhila Sekli-Belaidi, Gabriel Lemercier, Dodzi Zigah, Michel Rigoulet, Anne Devin, Néso Sojic, Pierre Temple-Boyer, Jérôme Launay, Stéphane Arbault

\section{To cite this version:}

Venkata Suresh Vajrala, Fadhila Sekli-Belaidi, Gabriel Lemercier, Dodzi Zigah, Michel Rigoulet, et al.. Microwell Array integrating Nanoelectrodes for Coupled Opto-electrochemical Monitorings of Single Mitochondria. Biosensors and Bioelectronics, 2019, 126, pp.672-678. 10.1016/j.bios.2018.11.036 . hal-02043423

\author{
HAL Id: hal-02043423 \\ https://hal.laas.fr/hal-02043423
}

Submitted on 21 Feb 2019

HAL is a multi-disciplinary open access archive for the deposit and dissemination of scientific research documents, whether they are published or not. The documents may come from teaching and research institutions in France or abroad, or from public or private research centers.
L'archive ouverte pluridisciplinaire HAL, est destinée au dépôt et à la diffusion de documents scientifiques de niveau recherche, publiés ou non, émanant des établissements d'enseignement et de recherche français ou étrangers, des laboratoires publics ou privés. 


\section{Microwell Array integrating Nanoelectrodes for Coupled Opto-electrochemical Monitorings of Single Mitochondria}

Venkata Suresh VAJRALA ${ }^{\mathrm{a}}$, Fadhila SEKLI BELAIDI ${ }^{\mathrm{b}}$, Gabriel LEMERCIER $^{\mathrm{b}}$, Dodzi ZIGAH ${ }^{\mathrm{a}}$, Michel RIGOULET ${ }^{\mathrm{c}}$, Anne DEVIN $^{\mathrm{c}}$, Neso SOJIC ${ }^{\mathrm{a}}$, Pierre TEMPLE-BOYER ${ }^{\mathrm{b}}$, Jérôme LAUNAY ${ }^{\mathrm{b}}$ and Stéphane ARBAULT ${ }^{\mathrm{a}}$

a. Univ. Bordeaux, ISM, CNRS UMR 5255, INP Bordeaux, Pessac, France

b. LAAS-CNRS, Université de Toulouse, F-31400 Toulouse, France

c. Univ. Bordeaux, IBGC, CNRS UMR 5095, Bordeaux, France

Corresponding author: Dr Stéphane Arbault; Univ. Bordeaux, ISM, CNRS UMR 5255, NSysA group, ENSCBP, Pessac, France; e-mail: stephane.arbault@u-bordeaux.fr 
Abstract: Chips composed of microwell arrays integrating nanoelectrodes (OptoElecWell) were developed to achieve dual optical and electrochemical detections on isolated biological entities. Each array consists in $10^{6}$ microwells of $6 \mu \mathrm{m}$ diameter $\times 5.2 \mu \mathrm{m}$ height each, with a transparent bottom surface for optical observations, a platinum nano-ring electrode at its halfheight for in situ electrochemistry, and a top open surface to inject solutions. Then, populations of individual mitochondria isolated from yeasts (Saccharomyces cerevisiae) were let to sediment on the array and be trapped within microwells. The trapping efficiency reached $20 \%$ but owing to the large number of microwells on the platform, hundreds of them could be filled simultaneously by single mitochondria. This allowed to follow up their individual energetic status based on fluorescence microscopy of their endogenous NADH. Simultaneously, the array of interconnected Pt nanoelectrodes in the microwells was used to monitor in situ variations of dioxygen consumed by all mitochondria captured in the device. Mitochondrial bioenergetics were modulated sequentially using respiratory chain-ATP synthase substrates (ethanol and ADP) and inhibitor (antimycin A). Overall, we show how two complementary analytical approaches, fluorescence and electrochemical detections, can be coupled for a multi-parametric monitoring of mitochondrial activities, with a resolution ranging from a small population (whole device) to the single mitochondrion level (unique well).

Keywords: Microdevice; Microwells; Nanoelectrode; Cyclic voltammetry; Fluorescence microscopy; Mitochondria; Oxygen; Bioenergetics 


\section{Introduction}

Mitochondria are the power factories of aerobic cells since they comprise the most prominent source of ATP, they are also involved in multiple anabolic and catabolic pathways. Due to genetic flaws and/or function alterations mitochondria play a central part in cellular ageing and in numerous diseases, including myopathies, cardiovascular disorders and cancers(Alston et al. 2017; Chinnery et al. 2012; Galluzzi et al. 2012; Gonzalez-Freire et al. 2015; Hauser and Hastings 2013; Wallace 2012; Yin et al. 2014). Mitochondria within a cell usually constitute a network with genetic and metabolic individual discrepancies and evolutions which increase along cell life (Chinnery et al. 2012; Dowling 2014; Kembro et al. 2013; Reiner et al. 2010; Youle and van der Bliek 2012). In other words, mitochondria are not all equivalent in terms of bioenergetics efficiency and status. This was until recently poorly taken into consideration for mitochondrial studies which are usually based on large populations of isolated entities or cells and diverse detection methods. Indeed, the most widely used techniques including fluorescence spectroscopy, chemiluminescence and electrochemistry (e.g. the Clark electrode) provide mean responses of mitochondria populations following bioenergetics activation/inhibition protocols (Allen et al. 2009; Dikalov and Harrison 2014; Hall et al. 2013; Quarato et al. 2011; Vasdekis and Stephanopoulos 2015; Zand et al. 2013). As a consequence, in order to decipher on mitochondrial heterogeneity and exact metabolic responses, studies at single organelle level have drawn huge interest (Appelhans and Busch 2017; Chang and Marshall 2017; Follain et al. 2017; Norregaard et al. 2017; Suraniti et al. 2013; Vajrala et al. 2014; Vajrala et al. 2016).

Among all available techniques, spectrophotometry and electrochemistry are the ones providing higher detection sensitivities while using limited amounts of mitochondria. Electrochemical methods are simple, sensitive, and provide real-time detection. Particularly, ultramicroelectrodes (UME) have led to numerous biomedical applications, since these micrometric-size electrodes sense concentration gradients of redox species within environments of a few tens of micrometers (pL volumes) (Amatore et al. 2008a; Amatore et al. 2008b; Ino et al. 2017; Youle and van der Bliek 2012; Zoski 2002). In addition, they offer advantageous features such as a true steady-state diffusion-limited currents, small ohmic drop $(I \times R)$ from solution resistance and short response times (low capacitive currents). They have thus been used to monitor responses from single cells (adrenal cells, neurons, fibroblasts, macrophages...) (Amatore et al. 2006; Amatore et al. 2008b; Amatore et al. 2010; Lin et al. 2018; Schulte and Schuhmann 2007). We and others have reported the real-time quantification of dioxygen consumption and hydrogen peroxide release by isolated mitochondria using amperometry with 
platinized UMEs (Ben-Amor et al. 2013; Ben-Amor et al. 2014; Marcu et al. 2012; Suraniti et al. 2014). However, in order to scale it down and target the single organelles, we have envisioned the use of nanoelectrodes (Clausmeyer and Schuhmann 2016; Fan et al. 2016; Karimian et al. 2017; Rassaei et al. 2014; Wang et al. 2012). The diffusion volume probed by a nanoelectrode would indeed be adequate for sensing the environment of a single mitochondrion, which characteristic size is between $300 \mathrm{~nm}$ to $1200 \mathrm{~nm}$.

On the other hand, fluorescence monitoring of single mitochondria evolved recently as a strategy for highly sensitive analyses of mitochondrial metabolic markers (Quarato et al. 2011; Suraniti et al. 2013; Vajrala et al. 2014; Vajrala et al. 2016; Zand et al. 2013). Markers include the intrinsic autofluorescent NADH and FAD metabolites, as well as extrinsic dyes (rhodamine derivatives) for the mitochondrial membrane potential $(\Delta \Psi)$, reactive oxygen species and other parameters (Kilgore et al. 2013; Li et al. 2016; Zhu et al. 2016). The microscopic platforms for single mitochondria monitoring are generally dependent on nanofluidics, nanohole arrays for trapping and surface functionalization for immobilization (Lim et al. 2012; Zand et al. 2013). Nevertheless, with such systems are certain restrictions such as an efficient delivery of oxygen and nutrients, a size dependent immobilization of mitochondria and the low number of entities measured. Lately, our work has been focused on microwell array strategy, which rely on random mitochondrial immobilization and do not require specific surface modifications. Platforms were obtained from etched optical fiber bundles and PDMS microwells, which both offer large scale studies of single mitochondria under bioenergetic activation and inhibition conditions (Suraniti et al. 2013; Vajrala et al. 2014; Vajrala et al. 2015; Vajrala et al. 2016).

Both above methodological families equally provide important information about mitochondrial metabolism, from either an external (electrochemistry) or internal (fluorescence) side. The goal of the present work was to develop platforms allowing these two techniques to run simultaneously on a same mitochondrial sample. A well-ordered microwell array (Belaidi et al. 2016) with integrated nanoelectrodes (OptoElecWell) was built over a transparent glass support. Based on silicium microtechnologies in conjunction with reactive ion etchings, we obtained opened microwells with ring nanoelectrodes inside each well.(Belaidi et al. 2016). The present work describes (i) the optimization of these OptoElecWell microdevices (ii) their efficient entrapment of single mitochondria and (iii) simultaneous electroanalysis of their oxygen consumption and fluorescence microscopy of individual NADH variations. The 
sensitivity and reliability of measurements was shown by the use of mitochondrial respiratory chain effectors.

\section{Materials and Methods}

\subsection{Preparation of mitochondria}

This work was based on mitochondria extracted from yeasts (Saccharomyces cerevisiae, Yeast Foam strain). Yeast culture, mitochondria isolation and conservation in frozen state (beads at $80^{\circ} \mathrm{C}$ ) were achieved as described previously (Suraniti et al. 2013; Vajrala et al. 2015).Mitochondria were analyzed in a specific medium, namely the Mitochondrial Buffer (MB) containing $0.63 \mathrm{M}$ deionized mannitol, $0.36 \mathrm{mM}$ EGTA, $10 \mathrm{mM}$ Tris-maleate, $5 \mathrm{mM}$ Tris-phosphate, adjusted at $\mathrm{pH} 6.8$.

\subsection{OptoElecWell microdevice fabrication}

Microdevice development was based on silicon microtechnologies.(Belaidi et al. 2016) Briefly, on a glass wafer (B33 type, $500 \mu \mathrm{m}$ thickness) were deposited $2.5 \mu \mathrm{m}$ thickness silicon oxide by plasma-enhanced chemical vapour deposition (PECVD), a $200 \mathrm{~nm}$ platinum layer in sandwitch between two $20 \mathrm{~nm}$-thick titanium adhesion layers, and another $2.5 \mu \mathrm{m} \mathrm{SiO}_{2}$ layer to form the $\mathrm{SiO}_{2} / \mathrm{Ti} / \mathrm{Pt} / \mathrm{Ti} / \mathrm{SiO}_{2}$ stacking. Through a patterned photoresist mask, inductively coupled plasma reactive ion etching (ICP-RIE) with three different gaseous mixtures $\left(\mathrm{CF}_{4} / \mathrm{Ar}\right.$, $\mathrm{SF}_{6}$ and $\mathrm{Cl}_{2}$ ) was used to etch the whole stack and fabricate microwells (diameter: $6 \mu \mathrm{m}$, depth : $5.2 \mu \mathrm{m}$ ) with integrated ring nanoelectrodes. At last, a $\mathrm{CF}_{4} / \mathrm{Ar}$ etching step was performed to contact electrically the nanoelectrode array.

\subsection{Electrochemical measurements}

The electrochemical measurements were carried out using a potentiostat (BioLogic Inc. VSP300, EC-Lab software) equipped with an ultralow current module. A freshly chlorinated silver wire and a platinum wire were used as the reference RE and counter CE electrodes, respectively, and placed within the solution on top of the microchip (nanoelectrode array as the working electrode WE).

\subsection{Fluorescence microscopy of mitochondria}

All experiments were performed at $25^{\circ} \mathrm{C}$ on the stage of an inverted microscope (Leica DMI 6000, Germany). Imaging was achieved with a 40× (N.A. 0.9) oil immersion objective, recorded by a camera (ORCA-Flash4.0 V2 SCMOS type, Hamamatsu, Japan) using a $200 \mathrm{~ms}$ 
exposure time without additional gain. Mitochondrial NADH was monitored by epifluorescence (Leica EL6000 source) with a DAPI-type filter (excitation and emission wavelengths are BP 340-380 nm and LP $425 \mathrm{~nm}$, respectively). Fluorescence intensities were measured (Metamorph, Molecular Devices, USA) by drawing regions of interest (line scan of 4-8 $\mu \mathrm{m}$ length) centered on each single mitochondrion spot. All intensities refer to the difference between maximum (mitochondrial fluorescence) and minimum (microwell background) values, meaning they were individually background-subtracted. Further statistical analyses of data were performed (Origin 8.0, OriginLab, USA).

\subsection{Opto-electrochemical measurements on mitochondria}

A PDMS (Poly-dimethylsiloxane, RTV615 kit, Momentive Performance Materials, USA) barricade (20 mm diameter x $3 \mathrm{~mm}$ height) was glued (CAF4 paster, Bluestar Silicon, USA) on top of the chip and let to dry. Before use, each OptoElecWell system was cleaned properly with water and ethanol, then treated by an oxygen plasma (Harrick Plasma cleaner) for 10 minutes. Meanwhile mitochondrial sample was defrozen and stored in an ice bath. The microdevice tank was filled with $500 \mu \mathrm{L}$ of buffer, then followed by addition of mitochondria solution $\left(1 \mathrm{mg} . \mathrm{mL}^{-}\right.$ $\left.{ }^{1}\right)$. The whole solution was homogenized very gently and let to rest in order for mitochondria to sediment progressively in the microwell array. Further, washings were performed by removing and adding at least four times $2 \mathrm{~mL}$ of buffer in the PDMS tank. All electrodes were connected to the potentiostat and the microscopy focus was adjusted to monitor mitochondria in the microwells. Ethanol (analytical grade), Adenosine diphosphate (ADP) and antimycin A (AMA) solutions were prepared in the mitochondrial buffer at following final concentrations: Ethanol $1 \% \mathrm{v} / \mathrm{v}, 2 \mathrm{mM}$ ADP and AMA $1.8 \mu \mathrm{M}$. For each bioenergetic stage, $100 \mu \mathrm{L}$ of stimulation-inhibition solution was introduced in the device tank.

\section{Results and Discussion}

\subsection{Design and optimization of OptoElecWell microdevices}

We have first designed microsystems allowing to isolate and observe single mitochondria while achieving simultaneously the electrochemical detection in situ and fluorescence spectrophotometry. Our past experience in single mitochondria monitoring by fluorescence microscopy on glass slides and PDMS surfaces, led us to design microwells organized in an array of at least hundreds of units, in order to gain a statistically significant number of mitochondria under analysis. The diameter and depth of microwells had to be sufficient to let mitochondria deposit and sediment within wells while enabling fast diffusion of solutions 
(activators/inhibitors). The distance between wells was also another key parameter since it should prevent the mixing of contents between adjacent microwells. In addition, even if a recessed feature favors confinement, ring nanoelectrodes have to be sufficiently separated one from the other to avoid any crosstalk between their diffusion layers (Godino et al. 2009; Huang et al. 2009; Zoski 2002).

Consequently, we have developed protocols to obtain a microwell array, on a transparent glass substrate, with individual diameters $\mathrm{D}$ of $6 \mu \mathrm{m}$ and of equivalent depth $(\mathrm{H}=5.2 \mu \mathrm{m})$ (roughly a unitary aspect ratio), and an inter-distance between them of $5 \times$ radius ( $\mathrm{R}$ ) to $20 \times \mathrm{R}$ (Figure 1 ). Each microwell must contain a working platinum ring nanoelectrode (RNE, $200 \mathrm{~nm}$ width) to detect local variations of mitochondrial metabolites $\left(\mathrm{O}_{2}\right.$ or $\left.\mathrm{H}_{2} \mathrm{O}_{2}\right)$. We have recently reported the protocols for the building of such microdevice (Belaidi et al. 2016). The microfabrication process is based on layer by layer deposits of: $\mathrm{SiO}_{2}(2.5 \mu \mathrm{m}$ mean thickness) / $\mathrm{Ti}(20 \mathrm{~nm}$ thickness as an adhesion layer) / $\mathrm{Pt}$ (200 nm thickness conductive layer) / $\mathrm{Ti}$ (20 nm) / $\mathrm{SiO}_{2}$ (2.5 $\mu \mathrm{m})$ over a transparent glass substrate (500 $\mu \mathrm{m}$ thickness). The microwells $(6 \pm 0.2 \mu \mathrm{m} \mathrm{D} \times 5.2$ $\pm 0.2 \mu \mathrm{m} \mathrm{H}$ ) were obtained by a multi-step reactive ion etching protocol providing $\mathrm{SiO}_{2}$ surfaces and flanks with low roughness and discovering efficiently the platinum surfaces (see images in Figure 1) (Li et al. 2003; Matsutani et al. 2012; Park et al. 2005).

a)
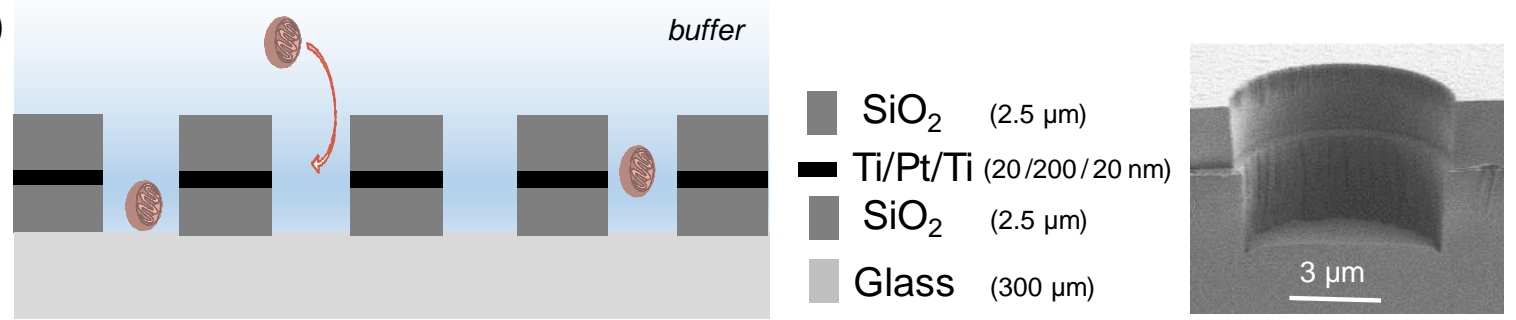

b)

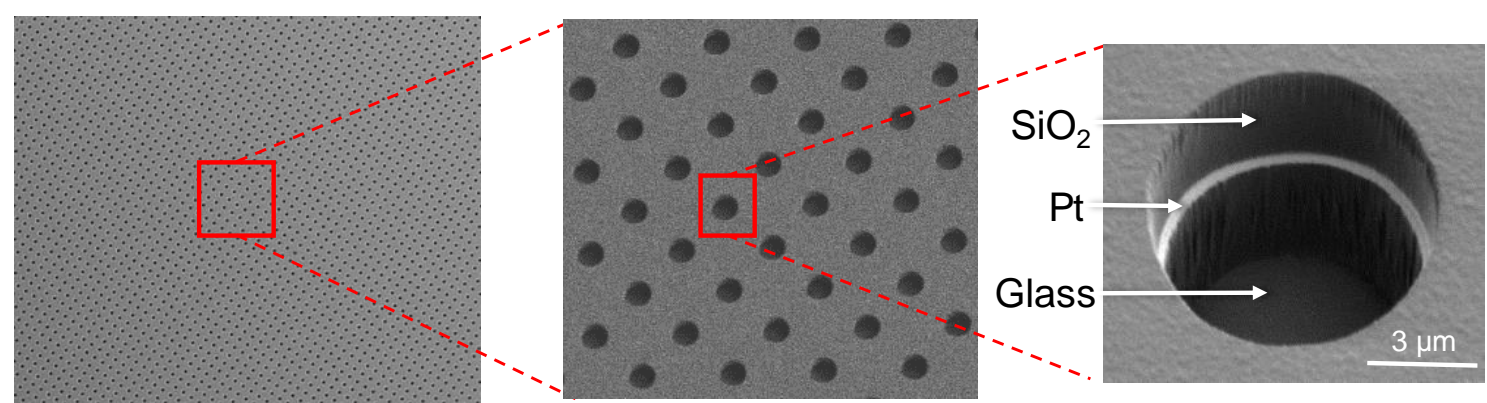

Figure 1- (a) left: Scheme of the OptoElecWell array for the monitoring of single mitochondria activities, right: Cross-sectional SEM view of a typical microwell; (b) SEM images of the $\mathrm{SiO}_{2}-$ based microwell array (mean diameter: $6 \mu \mathrm{m}$; mean depth: $5.2 \mu \mathrm{m}$ ) with integrated platinumring nanoelectrodes (thickness: $200 \mathrm{~nm}$ ) on a glass substrate. 
Because of material-dependent etching kinetics, platinum surfaces appeared as regular rings (200 nm thickness) with an exposed top border (300 nm width) within all wells. Multi-physic simulations were conducted to study the behavior of the recessed nanoelectrodes as a function of all geometric parameters.(Belaidi et al. 2016) The highest collection efficiency (nearly 90\%) is obtained for an aspect ratio $H / D \geq 1$, and for nanoelectrodes located at the mid-height of wells. Concerning the distance between wells, this parameter is critical for both the electrochemical detection and the entrapment of mitochondria. Indeed, while wells should be very close from each other (dense packing) in order to maximize their filling success rate, they should be distant enough (loose packing) so as to let nanoelectrodes work independently. Simulations and experiments have shown that quasi steady-state voltammograms were obtained (ferrocene-methanol and ferrocyanide redox probes) for a separation distance of $5 \times \mathrm{R}$ between wells whereas pure steady-state voltammograms were obtained for $20 \times \mathrm{R}$ value (Belaidi et al. 2016). As shown experimentally below, the dioxygen electrochemical detection in microwells will be achieved by fast cyclic voltammetry (scanning rate $\geq 1 \mathrm{~V} \cdot \mathrm{s}^{-1}$ ) in transient mode, which response was not dependent on device geometry. Consequently, when considering all constraints, OptoElecWell devices constituted of $10^{6}$ wells of $6 \mu \mathrm{m}(\mathrm{D}) \times 5.2 \mu \mathrm{m}(\mathrm{H})$, distant of $15 \mu \mathrm{m}(5 \times \mathrm{R})$, integrating the nanoelectrodes and external counter (millimetric Platinum wire) and reference $(\mathrm{Ag} / \mathrm{AgCl})$ electrodes in a PDMS reservoir on top, have been used for in vitro characterizations and further mitochondrial studies.

\subsection{Electrochemical responses of the OptoElecWell microdevices}

Platinum was chosen as the material for nanoelectrodes because of its wide electroactive window in water and since this is the most usual and efficient material for electrochemical oxygen sensing (generating water over a $4 \mathrm{e}^{-} / 4 \mathrm{H}^{+}$reaction), which is a major goal of this work. Electrochemical performances of the platinum nanoelectrodes within the microwell array were first characterized by cyclic voltammetry in a $0.1 \mathrm{M} \mathrm{H}_{2} \mathrm{SO}_{4}$ solution (see Figure $2 \mathrm{a}$ ). The voltammograms obtained from such thin layer platinum deposits depicts clear proton reduction waves and oxidation waves related to the formation of platinum oxides. It was manifest that the response of these platinum nanoelectrodes within microwells is very similar to the one of native solid electrodes and also demonstrates the electrochemical/structural quality of our fabrication subroutine to build up this integrated chip. 
a)

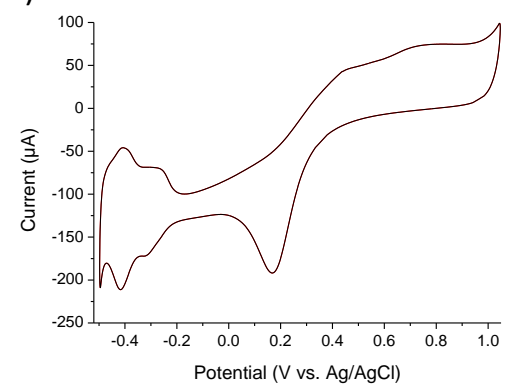

b)

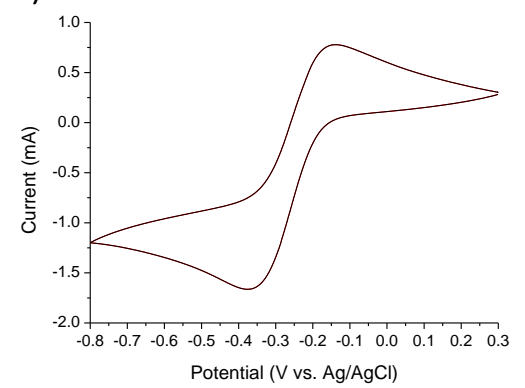

c)

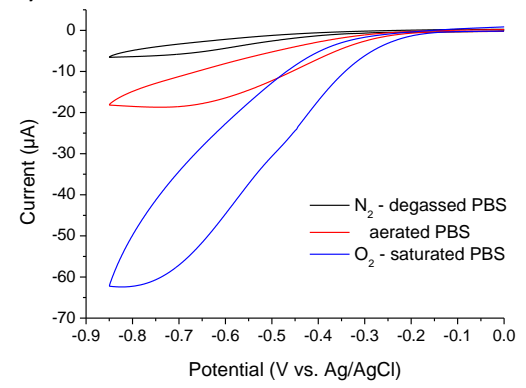

Figure 2- Electrochemical characterizations by cyclic voltammetry of the nanoelectrode array in the OptoElecWell microdevice: (a) voltammogram $\left(100 \mathrm{mV}^{-1}{ }^{-1}\right)$ recorded in a $0.1 \mathrm{M} \mathrm{H}_{2} \mathrm{SO}_{4}$ solution; (b) voltammogram $\left(10 \mathrm{mV} . \mathrm{s}^{-1}\right)$ recorded in a $3 \mathrm{mM} \mathrm{Ru}\left(\mathrm{NH}_{3}\right)_{6} \mathrm{Cl}_{3}$ solution in PBS buffer (pH 7.4, $10 \mathrm{mM})$; (c) voltammograms $\left(10 \mathrm{mV} . \mathrm{s}^{-1}\right)$ recorded in PBS buffer $(\mathrm{pH} 7.4,10$ $\mathrm{mM}$ ) containing different oxygen concentrations. $\mathrm{An} \mathrm{Ag} / \mathrm{AgCl}$ reference electrode and a platinum wire used as a counter electrode were both placed in the bulk solution on top of the chip ( $2 \mathrm{~mL}$ volume).

Further characterizations were achieved in aqueous buffer solutions, PBS (pH 7.4) and a specific buffer for mitochondrial experiments ( $\mathrm{pH}$ 6.8, see experimental section). With a classical chemical redox probe in PBS, namely ruthenium hexaamine, a quasi-reversible reduction wave was observed with a pseudo steady-state behavior at low scan rate $\left(10 \mathrm{mV} . \mathrm{s}^{-1}\right)$ and peak-shaped voltammograms at higher scan rates $\left(\geq 100 \mathrm{mV} \cdot \mathrm{s}^{-1}\right)$. Pure steady-state voltammograms could not be obtained even at very low scan rates (about $1 \mathrm{mV} . \mathrm{s}^{-1}$ ). This is due to the recessed position of nanoelectrodes within microwells and to their inter-distance, which induces a diffusion field intermediate between planar and hemi-spherical geometries (Guo and Lindner 2009; Ma et al. 2013). Consequently, voltammograms shape as pseudo steady-state responses. The next goal was to evaluate the oxygen sensing ability of the device. Electrochemical reduction of dioxygen was performed in PBS under various concentrations (Figure 2c). The oxygen reduction was well detected with a plateau current reached at about $0.75 \mathrm{~V}$ vs $\mathrm{Ag} / \mathrm{AgCl}$. The sensitivity of the nanoelectrode array to $\mathrm{O}_{2}$ concentration variations was excellent and fairly comparable to a usual platinum Clark electrode. Nevertheless, when achieving multiple scans, we observed a progressive loss of amplitude of the reduction wave, showing that oxygen is consumed faster within microwells ( $0.14 \mathrm{pL}$ mean individual volume) than it diffuses from the solution. This meant that the time-scale of measurements should be adapted in order to not consume oxygen significantly and deprive mitochondria from their 
substrate. In further experiments, cyclic voltammograms were consequently performed at faster scan rates, from 1 to 4 V.s. ${ }^{-1}$, with a delay of 10 to 20 seconds between scans (see part 3.4).

\subsection{Entrapment of single mitochondria in microwells}

OptoElecWell microdevices are dense microwell arrays, e.g. 2500 microwells $/ \mathrm{mm}^{2}$, for the high throughput entrapment and metabolic monitoring of single mitochondria. The handling of solutions in the device was achieved by adding a $5 \mathrm{~mm}$-high PDMS tank on the upper side of the chip, while keeping the glass bottom surface open for microscopy (Figure 3a). The tank contained $2 \mathrm{~mL}$ of buffer solution above microwells, preventing evaporation and the lack of oxygen for mitochondria. The device was pre-treated by an oxygen plasma to improve hydrophilicity of surfaces (Jacobs et al. 2012; Jokinen et al. 2012). We observed a rapid and homogenous filling of microwells by aqueous solutions. Then, we added a suspension of mitochondria ( $\left.1 \mathrm{mg} \cdot \mathrm{mL}^{-1}\right)$ in the tank and observed their appearance in the microwells by microscopy. Observations could be performed in transmission mode with white light but this mode did not allow to easily differentiate between mitochondria and possible protein or membrane aggregates. Epi-fluorescence observation helped better to define the localization of mitochondria, thanks to their NADH content. First observations depicted strong fluorescence responses in most of microwells (left image of Figure 3b). This was due to the high density of mitochondria into and also on top of the microwells. The OptoElecWell were then subjected to careful washings with buffer in order to eliminate efficiently the excess of mitochondria. Under these conditions, the mean trapping efficiency of individual mitochondria was $18 \%$, while a few more additionally contained several mitochondria (2-4 entities). When applying less washing steps, one may reach a higher trapping efficiency in microwells (about 50\%), but with multiple mitochondria in each (typically 1 to 5 entities). This situation corresponding to the analysis of small populations would lead to higher responses with both analytical methods (see below), but would escape from the single mitochondrion criterion targeted herein. 


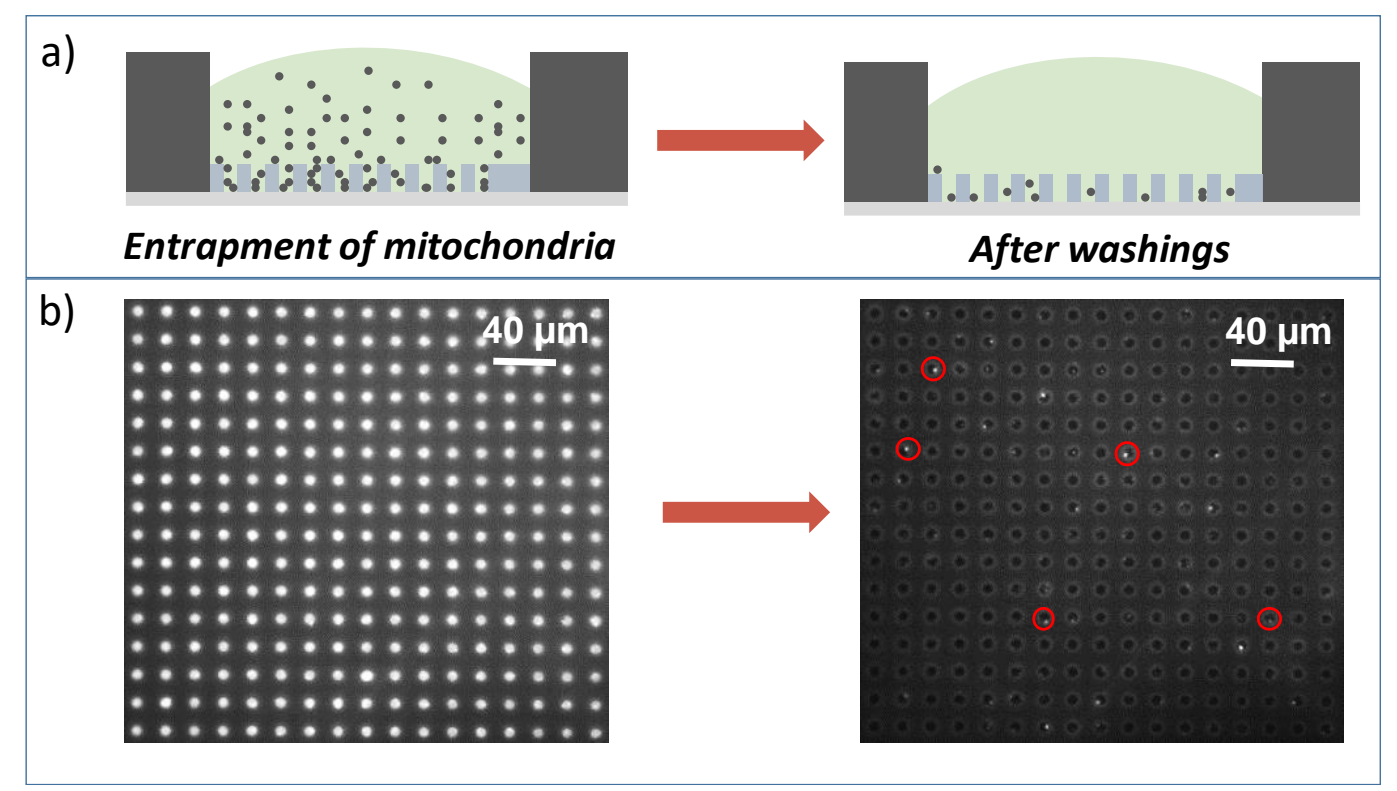

Figure 3- (a) Schematic representation of the entrapment of single mitochondria within the OptoElecWell device starting from a suspension of mitochondria in buffer and following several washing steps; (b) Optical imaging of the evolution of mitochondria presence in microwells before and after washings with the buffer solution. Observations are based on mitochondrial NADH fluorescence microscopy (40× objective). Red circles show up some entrapped single mitochondria, displaying different sizes and intensities.

\subsection{Combined optical and electrochemical monitoring of mitochondrial responses}

The simultaneous sensing of mitochondrial activities by combined electrochemistry and fluorescence spectrophotometry was based on the monitoring of two essential substrates of the respiratory chain: the endogenous $\mathrm{NADH}$ (fluorescence) and dioxygen (electrochemical). NADH displays a fluorescence emission (peak at $460 \mathrm{~nm}$ ), while the oxidized form $\mathrm{NAD}^{+}$does not fluoresce. NADH is the substrate of complex I and of internal NADH dehydrogenases in mammalian and yeast mitochondria (Saccharomyces cerevisiae used here), respectively. Its steady-state concentration was estimated as a fraction of a millimolar (Bakker et al. 2001; Talbot et al. 2007). Mitochondrial oxygen consumption has also been recognized traditionally as the key parameter for assessing the respiratory chain efficacy. The most efficient approach for oxygen sensing is the classic Clark electrode based on a platinum working electrode, and we have demonstrated in section 3.2 that this can also be performed with OptoElecWell platforms. 
The microscope imaging (40x objective) of the array provided the simultaneous observation of about 400 microwells, and the device was not displaced during the whole experiment (20-30 min.) in order not to modify the focus and provoke mechanical movements. Once mitochondria were let to sediment in the microwells, their individual NADH fluorescence were measured every minute by using region of interest patterns (around each microwell containing a single entity), at different bioenergetics states following additions of respiratory substrates and inhibitors. Figure 4 shows the mean variations of 75 individual mitochondrial responses measured over 3 different experiments. Only a few mitochondria (20\% at most) were not responding favorably to activators/inhibitors, as observed in our previous studies (Suraniti et al. 2013; Vajrala et al. 2016).
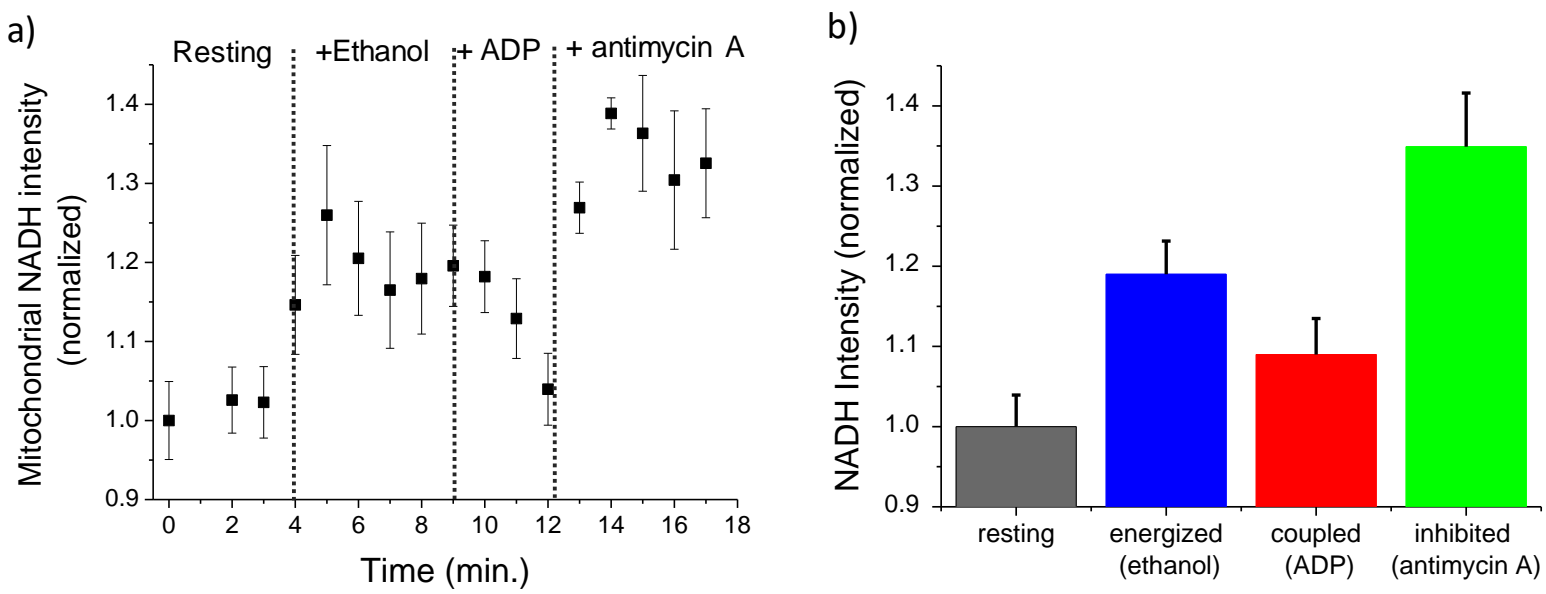

Figure 4- (a) Fluorescence monitoring along the bioenergetic modulations of NADH fluorescence in individual mitochondria entrapped within OptoElecWell. Each point is a mean response ( $\mathrm{n}=75$ mitochondria from 3 different experiments) \pm SEM at each time of measurement. All values were normalized versus their initial one at resting stage (no activation or inhibition). (b) Comparison of the mean values at each stage, i.e. following injections of $1 \%$ ethanol, $2 \mathrm{mM}$ ADP and $1.8 \mu \mathrm{M}$ antimycin A solutions, respectively.

Meanwhile, electrochemical measurements were conducted at the nanoelectrode array in the microsystem. As mentioned earlier, a sampling detection method is necessary to sense rapidly and at regular times the local oxygen concentration variations next to mitochondria, without modifying substantially this concentration $(220 \mu \mathrm{M}$ at room temperature and normal atmosphere) or possibly modifying also the local $\mathrm{pH}$. We used fast cyclic voltammetry $(\mathrm{CV}$, scan rate of 1 to $4 \mathrm{~V} \cdot \mathrm{s}^{-1}$, with a delay of 10 to 20 seconds between scans) for measuring $\mathrm{O}_{2}$ 
variations in microwells. CV was preferred to other methods since it constantly informs on the reduction processes involved, at least the $\mathrm{O}_{2}$ reduction wave evolution (Figure 5). Repetitive scans were applied at the nanoelectrodes for at least $20 \mathrm{~min}$. before launching mitochondrial measurements. The CV shape was fairly stable and the amplitude started to decrease slightly when mitochondria were injected and present into microwells. Though the number of mitochondria under observation was only a few tens, the whole microsystem was much larger in dimensions and we estimated that about 50000 mitochondria participated to dioxygen concentration decrease.
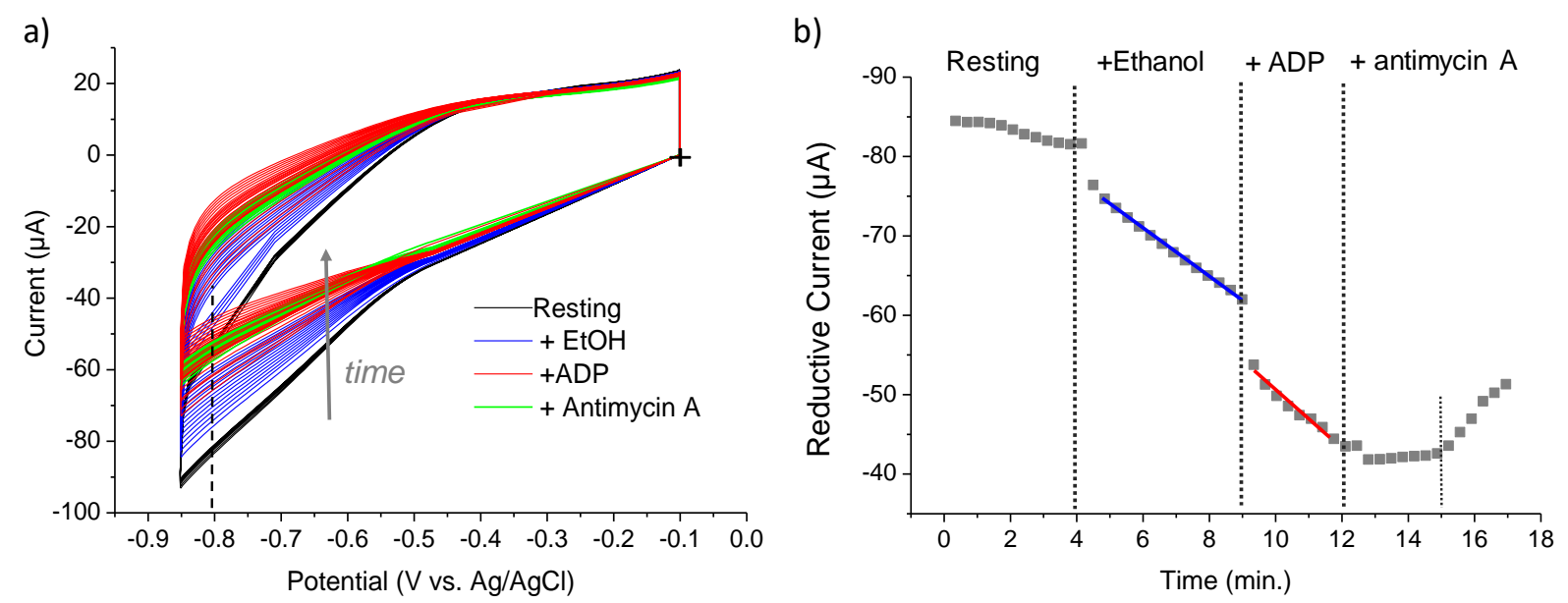

Figure 5- (a) Electrochemical monitoring of the oxygen consumption by mitochondria present in the OptoElecWell device under different stages (Scan rate: $4 \mathrm{~V} \cdot \mathrm{s}^{-1}$, delay of $10 \mathrm{sec}$. between scans). (b) Evolution of the reduction current at $-0.8 \mathrm{~V}$ vs $\mathrm{Ag} / \mathrm{AgCl}$ measured on each scan, following additions of bioenergetic substrates (Ethanol, ADP) and an inhibitor (antimycin A). Measurements were conducted simultaneously to NADH ones reported in Figure 4.

Then, ethanol (1\% V/V in buffer) was injected in the buffer solution surrounding the array. Ethanol (EtOH) is employed as an energetic substrate since it is converted in Saccharomyces cerevisiae mitochondria into acetaldehyde and NADH by alcohol dehydrogenases. NADH induces respiratory chain functioning (non-phosphorylating condition) and consumption of dioxygen, as monitored by OptoElecWell, i.e. a $19 \%$ increase of NADH (figure 4b) and a decrease of 12-15 $\mu \mathrm{M} \mathrm{O}_{2} \cdot \mathrm{min}^{-1}$ local oxygen concentration (negative slope of $2.5 \mathrm{nA} \cdot \mathrm{min}^{-1}$ in figure $5 \mathrm{~b}$ ). After $5 \mathrm{~min}$., the microsystem was provided with additional $2 \mathrm{mM}$ adenosinediphosphate (ADP). ADP is the substrate of mitochondrial ATP synthases and induces the coupling of phosphorylation (ATP formation) and respiratory activities (OXPHOS), therefore 
increasing the rate of oxygen consumption (3.5 nA.min ${ }^{-1}$ slope, corresponding to $17-20 \mu \mathrm{M}$ $\mathrm{O}_{2} \cdot \mathrm{min}^{-1}$ ) by a factor of " 1.4 " (figure $5 \mathrm{~b}$ ). This value quoted as the mitochondrial respiratory control-ratio, was measured for a minute population of mitochondria $\left(5 \times 10^{4}\right)$ in microwells, and is in good agreement with conventional studies with the same mitochondria (Beauvoit et al. 1993; Ben-Amor et al. 2013; Suraniti et al. 2014). However, the usual quantities involved for oxygraphy measurements are milligrams of proteins, meaning $10^{6}-10^{8}$ mitochondria. On the other hand, the addition of ADP increases the rate of NADH consumption in functioning mitochondria, which caused NADH fluorescence to decrease progressively (Figure 4a). Finally, antimycin A, an inhibitor of complex III of the respiratory chain, was added to lead to the blockade of oxygen consumption and to a decrease of NADH consumption, inducing herein a $35 \%$ increment of mean NADH fluorescence compared to the resting stage (Figure 4a and 4b), and a progressive increase of oxygen concentration in microwells (see the positive slope on the graph after 15 minutes in Figure 5b) associated with the balancing of diffusion from the solution. All these results obtained with OptoElecWell microdevices are in excellent agreement with our previous works with fluorescence and electrochemical measurements performed separately on mitochondria from the same origin (Ben-Amor et al. 2014; Suraniti et al. 2014; Suraniti et al. 2013; Vajrala et al. 2016).

From a spectrophotometric perspective, OptoElecWell platforms allow to evaluate and accurately compare mean mitochondrial NADH intensities, but it is likewise possible to trace single mitochondria variations and point out specific mitochondrial populations exhibiting different activities and trends compared to the mean values. Figure S1 presents the histograms of value distributions in the four bioenergetics stages (Stage 1 to 4 ) and scatter plots describing $\mathrm{NADH}$ intensity increments/decrements in energized, phosphorylating and respiratory inhibited stages when compared to the previous stage. From figure S1a, we observe that individual values are showing general trends (higher or lower intensities) in line with the mean values (Figure $4 \mathrm{~b}$ ), but the sum of increment or decrement can be very different from one mitochondrion to the other (heterogenic nature). This observation is clearly in full agreement with our previous studies (Suraniti et al. 2013; Vajrala et al. 2016), nevertheless the higher interest of OptoElecWells resides in the simultaneous usage of fluorescence imaging and electrochemical sensing which eventually leads to the dynamic monitoring at least of two mitochondrial metabolic parameters.

\section{Conclusion}


We designed, developed and characterized a microdevice based on a microwell array integrating an optical window and ring-type Pt nanoelectrodes. We demonstrated how such microwell array could be employed for the entrapment of single mitochondria and provided a proof-of-concept study on simultaneous dual opto-electrochemical monitoring of bioenergetic activities: electroanalysis of oxygen consumption on a small population $\left(\sim 5 \times 10^{4}\right)$ and fluorescence microscopy to follow up endogenous NADH at single organelle level. The current work will open new opportunities in line with sensitive multi-parametric measurements of mitochondrial redox status in diverse physio-pathologic dysfunctions, including the Warburg effect in cancer cells, myopathies or degenerative processes of neurons.

\section{Acknowledgments}

This project was financially supported by the CNRS ("Centre National de la Recherche Scientifique “: UPR 8001, UMR 5255 and UMR 5095), University of Bordeaux, INP Bordeaux, and the ANR ("Agence Nationale pour la Recherche", project NANOMITO $\mathrm{n}^{\circ}$ ANR2011BSV502501). The technological realisations and associated research works were partly supported by the French RENATECH network. Bertrand Goudeau and Nicole Avéret are acknowledged for help during yeast culture and mitochondria preparations.

\section{Declaration of conflict of interest}

The authors declare no conflict of interest.

\section{References}

Allen, P.B., Doepker, B.R., Chiu, D.T., 2009. Analytical Chemistry 81(10), 3784-3791.

Alston, C.L., Rocha, M.C., Lax, N.Z., Turnbull, D.M., Taylor, R.W., 2017. Journal of Pathology 241(2), 236-250.

Amatore, C., Arbault, S., Bouret, Y., Cauli, B., Guille, M., Rancillac, A., Rossier, J., 2006. ChemPhysChem 7(1), 181-187.

Amatore, C., Arbault, S., Bouton, C., Drapier, J.C., Ghandour, H., Koh, A.C.W., 2008a. Chembiochem 9(9), 1472-1480.

Amatore, C., Arbault, S., Guille, M., Lemaitre, F., 2008b. Chemical Reviews 108(7), 25852621.

Amatore, C., Arbault, S., Koh, A.C.W., 2010. Analytical Chemistry 82(4), 1411-1419.

Appelhans, T., Busch, K.B., 2017. Biophysical reviews 9(4), 345-352.

Bakker, B.M., Overkamp, K.M., van Maris, A.J.A., Kotter, P., Luttik, M.A.H., van Dijken, J.P., Pronk, J.T., 2001. Fems Microbiology Reviews 25(1), 15-37.

Beauvoit, B., Rigoulet, M., Bunoust, O., Raffard, G., Canioni, P., Guerin, B., 1993. European Journal of Biochemistry 214(1), 163-172.

Belaidi, F.Sekli., Vanhove, E., Tiddi, W., Polverel, M., Lemercier, G., Lecestre, A., Dubreuil, P., Launay, J., Arbault, S., Temple-Boyer, P., 2016. Sensors and Actuators B-Chemical 232, 345-356. 
Ben-Amor, S., Devin, A., Rigoulet, M., Sojic, N., Arbault, S., 2013. Electroanalysis 25(3), 656663.

Ben-Amor, S., Vanhove, E., Belaidi, F.S., Charlot, S., Colin, D., Rigoulet, M., Devin, A., Sojic, N., Launay, J., Temple-Boyer, P., Arbault, S., 2014. Electrochimica Acta 126, 171-178.

Chang, A.Y., Marshall, W.F., 2017. Journal of Cell Science 130(5), 819-826.

Chinnery, P.F., Elliott, H.R., Hudson, G., Samuels, D.C., Relton, C.L., 2012. International Journal of Epidemiology 41(1), 177-187.

Clausmeyer, J., Schuhmann, W., 2016. Trac-Trends in Analytical Chemistry 79, 46-59.

Dikalov, S.I., Harrison, D.G., 2014. Antioxidants \& Redox Signaling 20(2), 372-382.

Dowling, D.K., 2014. Biochimica Et Biophysica Acta-General Subjects 1840(4), 1393-1403.

Fan, Y., Han, C., Zhang, B., 2016. Analyst 141(19), 5474-5487.

Follain, G., Mercier, L., Osmani, N., Harlepp, S., Goetz, J.G., 2017. Journal of Cell Science 130(1), 23-38.

Galluzzi, L., Kepp, O., Trojel-Hansen, C., Kroemer, G., 2012. Circulation Research 111(9), 1198-1207.

Godino, N., Borrise, X., Xavier Munoz, F., Javier del Campo, F., Compton, R.G., 2009. Journal of Physical Chemistry C 113(25), 11119-11125.

Gonzalez-Freire, M., de Cabo, R., Bernier, M., Sollott, S.J., Fabbri, E., Navas, P., Ferrucci, L., 2015. Journals of Gerontology Series a-Biological Sciences and Medical Sciences 70(11), 1334-1342.

Guo, J., Lindner, E., 2009. Journal Of Electroanalytical Chemistry 629(1-2), 180-184.

Hall, A., Larsen, A.K., Parhamifar, L., Meyle, K.D., Wu, L.-P., Moghimi, S.M., 2013. Biochimica Et Biophysica Acta-Bioenergetics 1827(10), 1213-1225.

Hauser, D.N., Hastings, T.G., 2013. Neurobiology of Disease 51, 35-42.

Huang, X.-J., O'Mahony, A.M., Compton, R.G., 2009. Small 5(7), 776-788.

Ino, K., Shiku, H., Matsue, T., 2017. Current Opinion in Electrochemistry 5(1), 146-151.

Jacobs, T., Morent, R., De Geyter, N., Dubruel, P., Leys, C., 2012. Plasma Chemistry and Plasma Processing 32(5), 1039-1073.

Jokinen, V., Suvanto, P., Franssila, S., 2012. Biomicrofluidics 6(1), 016501-01650110.

Karimian, N., Moretto, L.M., Ugo, P., 2017. Sensors 17(1), 65-90.

Kembro, J.M., Aon, M.A., Winslow, R.L., O'Rourke, B., Cortassa, S., 2013. Biophysical Journal 104(2), 332-343.

Kilgore, J.A., Dolman, N.J., Davidson, M.W., 2013. Current protocols in cytometry 66, Unit 12.31.-Unit 12.31.

Li, L., Abe, T., Esashi, M., 2003. Journal of Vacuum Science \& Technology B 21(6), 25452549.

Li, Y., Lu, Z., Liu, M., Xing, G., 2016. Chinese Journal of Organic Chemistry 36(5), 962-975. Lim, T.S., Davila, A., Zand, K., Wallace, D.C., Burke, P.J., 2012. Lab on a Chip 12(15), 27192725.

Lin, T.E., Rapino, S., Girault, H.H., Lesch, A., 2018. Chemical Science 9(20), 4546-4554.

Ma, C., Contento, N.M., Gibson, L.R., II, Bohn, P.W., 2013. Analytical Chemistry 85(20), 9882-9888.

Marcu, R., Rapino, S., Trinei, M., Valenti, G., Marcaccio, M., Pelicci, P.G., Paolucci, F., Giorgio, M., 2012. Bioelectrochemistry 85, 21-28.

Matsutani, A., Hayashi, M., Morii, Y., Nishioka, K., Isobe, T., Nakajima, A., Matsushita, S., 2012. Japanese Journal of Applied Physics 51(9), 098002.

Norregaard, K., Metzler, R., Ritter, C.M., Berg-Sorensen, K., Oddershede, L.B., 2017. Chemical Reviews 117(5), 4342-4375.

Park, J.H., Lee, N.E., Lee, J., Park, J.S., Park, H.D., 2005. Microelectronic Engineering 82(2), 119-128.

Quarato, G., Piccoli, C., Scrima, R., Capitanio, N., 2011. Mitochondrion 11(5), 764-773. 
Rassaei, L., Mathwig, K., Kang, S., Heering, H.A., Lemay, S.G., 2014. Acs Nano 8(8), 82788284.

Reiner, J.E., Kishore, R.B., Levin, B.C., Albanetti, T., Boire, N., Knipe, A., Helmerson, K., Deckman, K.H., 2010. Plos One 5(12), e14359.

Schulte, A., Schuhmann, W., 2007. Angewandte Chemie-International Edition 46(46), 87608777.

Suraniti, E., Ben-Amor, S., Landry, P., Rigoulet, M., Fontaine, E., Bottari, S., Devin, A., Sojic, N., Mano, N., Arbault, S., 2014. Angewandte Chemie-International Edition 53(26), 6655-6658. Suraniti, E., Vajrala, V.S., Goudeau, B., Bottari, S.P., Rigoulet, M., Devin, A., Sojic, N., Arbault, S., 2013. Analytical Chemistry 85(10), 5146-5152.

Talbot, J., Barrett, J.N., Barrett, E.F., David, G., 2007. Journal of Physiology-London 579(3), 783-798.

Vajrala, V.S., Suraniti, E., Garrigue, P., Goudeau, B., Rigoulet, M., Devin, A., Sojic, N., Arbault, S., 2014. Analytical and Bioanalytical Chemistry 406(4), 931-941.

Vajrala, V.S., Suraniti, E., Goudeau, B., Sojic, N., Arbault, S., 2015. Methods in molecular biology (Clifton, N.J.) 1264, 47-58.

Vajrala, V.S., Suraniti, E., Rigoulet, M., Devin, A., Sojic, N., Arbault, S., 2016. Integrative Biology 8(8), 836-843.

Vasdekis, A.E., Stephanopoulos, G., 2015. Metabolic Engineering 27, 115-135.

Wallace, D.C., 2012. Nature Reviews Cancer 12(10), 685-698.

Wang, Y.X., Noel, J.M., Velmurugan, J., Nogala, W., Mirkin, M.V., Lu, C., Collignon, M.G., Lemaitre, F., Amatore, C., 2012. Proceedings of the National Academy of Sciences of the United States of America 109(29), 11534-11539.

Yin, F., Boveris, A., Cadenas, E., 2014. Antioxidants \& Redox Signaling 20(2), 353-371.

Youle, R.J., van der Bliek, A.M., 2012. Science 337(6098), 1062-1065.

Zand, K., Pham, T., Davila, A., Wallace, D.C., Burke, P.J., 2013. Analytical Chemistry 85(12), 6018-6025.

Zhu, H., Fan, J., Du, J., Peng, X., 2016. Accounts of Chemical Research 49(10), 2115-2126.

Zoski, C.G., 2002. Electroanalysis 14(15-16), 1041-1051. 